\title{
ARTICLE Inhibition of drug-metabolizing enzymes by Jingyin granules: implications of herb-drug interactions in antiviral therapy
}

\author{
Feng Zhang ${ }^{1}$, Wei Liu ${ }^{2}$, Jian Huang ${ }^{1,3}$, Qi-long Chen ${ }^{1}$, Dan-dan Wang ${ }^{4}$, Li-wei Zou ${ }^{1}$, Yong-fang Zhao ${ }^{1,2}$, Wei-dong Zhang ${ }^{1}$,
} Jian-guang $\mathrm{Xu}^{1}$, Hong-zhuan $\mathrm{Chen}^{1}$ and Guang-bo $\mathrm{Ge}^{1}$

\begin{abstract}
Jingyin granules, a marketed antiviral herbal medicine, have been recommended for treating $\mathrm{H} 1 \mathrm{~N} 1$ influenza A virus infection and Coronavirus disease 2019 (COVID-19) in China. To fight viral diseases in a more efficient way, Jingyin granules are frequently coadministered in clinical settings with a variety of therapeutic agents, including antiviral drugs, anti-inflammatory drugs, and other Western medicines. However, it is unclear whether Jingyin granules modulate the pharmacokinetics of Western drugs or trigger clinically significant herb-drug interactions. This study aims to assess the inhibitory potency of the herbal extract of Jingyin granules (HEJG) against human drug-metabolizing enzymes and to clarify whether HEJG can modulate the pharmacokinetic profiles of Western drug(s) in vivo. The results clearly demonstrated that HEJG dose-dependently inhibited human CES1A, CES2A, CYPs1A, $2 \mathrm{~A} 6,2 \mathrm{C} 8,2 \mathrm{C} 9,2 \mathrm{D} 6$, and 2E1; this herbal medicine also time- and NADPH-dependently inhibited human CYP2C19 and CYP3A. In vivo tests showed that HEJG significantly increased the plasma exposure of lopinavir (a CYP3A-substrate drug) by 2.43 -fold and strongly prolonged its half-life by 1.91 -fold when HEJG $(3 \mathrm{~g} / \mathrm{kg})$ was co-administered with lopinavir to rats. Further investigation revealed licochalcone A, licochalcone B, licochalcone C and echinatin in Radix Glycyrrhizae, as well as quercetin and kaempferol in Folium Llicis Purpureae, to be time-dependent CYP3A inhibitors. Collectively, our findings reveal that HEJG modulates the pharmacokinetics of CYP substrate-drug(s) by inactivating CYP3A, providing key information for both clinicians and patients to use herb-drug combinations for antiviral therapy in a scientific and reasonable way.
\end{abstract}

Keywords: herbal extract of Jingyin granules (HEJG); cytochrome P450 enzymes (CYPs/P450s); herb-drug interactions (HDIs); CYP3A substrate-drugs

Acta Pharmacologica Sinica (2022) 43:1072-1081; https://doi.org/10.1038/s41401-021-00697-2

\section{INTRODUCTION}

Infectious diseases caused by viruses, including influenza, Ebola, and Coronavirus disease 2019 (COVID-19), have had significantly negative effects on human health, economic growth, and social stability [1-3]. Various therapeutic agents (such as antiviral drugs, anti-inflammatory drugs, immunosuppressive agents, and other Western medicines) have been developed and used for treating viral infectious diseases [4-6]. However, to fight against viral diseases in a more efficient way, drug-drug or herb-drug combinations are frequently used in antiviral therapy in clinical settings [7-9]. In China, several herbal medicines and Chinese medicine compound formulas (such as Banlangen granules, Jingyin granules, and Lianhua Qingwen capsules) have been validated as having antiviral activities against a range of viruses and have been recommended for combating a panel of viral diseases, including $\mathrm{H} 1 \mathrm{~N} 1$ influenza $A$ virus (H1N1) infection and COVID-19 [10-12]. In most cases, these antiviral Chinese medicines (CMs) are often co-administered with a variety of antiviral agents (such as oseltamivir and lopinavir) as antiviral therapy $[13,14]$. However, concomitant use of antiviral CMs with
Western drugs may trigger clinically relevant herb-drug interactions (HDIs) or adverse drug reactions; thus, it is urgent and necessary to assess the potential risks of HDls in antiviral therapy.

Jingyin granules, a marketed antiviral herbal product derived from an inhospital preparation of Shuguang Hospital (Shanghai, China), have been used in clinical settings for more than 40 years. Clinical applications have demonstrated that Jingyin granules have good curative effects for treating lung meridian wind heat syndrome and respiratory diseases with fever, sore throat, and cough $[15,16]$. Jingyin granules have been found to have antiviral activities against a range of viruses both in vitro and in vivo, and this herb is now recommended as a reserve medicine for preventing and treating $\mathrm{H} 1 \mathrm{~N} 1$ infection and COVID-19 in Shanghai. Jingyin granules are prepared from nine herbal medicines, including Herba Schizonepetae (Jingjie), Flos Lonicerae (Jinyinhua), Folium Llicis Purpureae (Sijiqing), Herba Houttuyniae (Yuxingcao), Folium Isatidis (Daqingye), Herba Taraxaci (Pugongying), Fructus Arctii (Niubangzi), Radix Ledebouriellae (Fangfeng), and Radix Glycyrrhizae (Gancao). Most of these herbs are reported

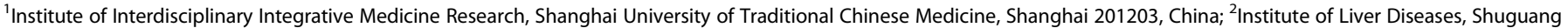

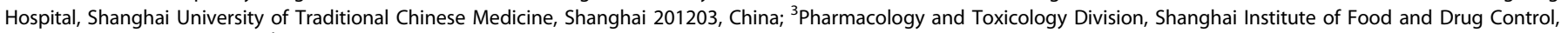
Shanghai 201203, China and ${ }^{4}$ SPH Xing Ling Sci. \& Tech. Pharmaceutical Co., Ltd, Shanghai 201703, China

Correspondence: Hong-zhuan Chen (hongzhuan_chen@hotmail.com) or Guang-bo Ge (geguangbo@dicp.ac.cn)

These authors contributed equally: Feng Zhang, Wei Liu

Received: 3 December 2020 Accepted: 12 May 2021

Published online: 28 June 2021 
to have antiviral and anti-inflammatory effects [17-24]. As a combination of nine herbs, Jingyin granules are composed of hundreds of natural constituents, some of which may strongly interact with a range of human drug-metabolizing enzymes (DMEs) or drug transporters and modulate the pharmacokinetics of co-administered therapeutic agents $[25,26]$. Because most marketed antiviral agents (such as oseltamivir, remdesivir, and lopinavir) are substrates of phase I DMEs [27-29], including human cytochrome P450 enzymes (CYPs or P450s) and carboxylesterases (CESs), the inhibitory or inactivating effects of Jingyin granules on these key DMEs as well as potential risks of HDls between Jingyin granules and antiviral agents should be carefully investigated.

This study aimed to assess the inhibition/inactivation potential of an herbal extract of Jingyin granules (HEJG) against human phase I DMEs and potential in vivo HDls between Jingyin granules and clinically used antiviral agent(s). Based on a panel of inhibition assays in vitro, HEJG dose-dependently inhibited human CES1A, CES2A, CYPs1A, 2A6, 2C8, 2C9, 2D6, and 2E1 and time- and NADPH-dependently inhibited CYP2C19 and CYP3A. Further investigation showed that HEJG also inhibited rat Cyp3a in a time-dependent manner. These in vitro data encouraged us to examine in vivo pharmacokinetic interactions between HEJG and CYP3A substrate-drug(s) by using rats as a surrogate model. The results clearly showed that HEJG significantly modulated the pharmacokinetic behavior of lopinavir (a CYP3A-substrate drug) when HEJG $(3 \mathrm{~g} / \mathrm{kg})$ was co-administered with lopinavir (160 mg/ $\mathrm{kg}$ ) in rats. A more in-depth investigation revealed that both Radix Glycyrrhizae and Folium Llicis Purpureae and their constituents triggered significant loss of CYP3A activity in time-, dose- and $\mathrm{NADPH}$-dependent manners, suggesting that these two herbs are responsible for CYP3A inactivation. All these observations offer new insight into $\mathrm{HDI}$ between Jingyin granules and antiviral agents. The key findings presented herein will be very helpful for guiding clinicians and patients to use the combination of Jingyin granules and antiviral agent(s) in a more scientific and reasonable way.

\section{MATERIALS AND METHODS}

Chemicals, reagents, and biological samples

HEJG (Lot No. 200203S) and the extract from nine individual herbs for preparing Jingyin granules (the preparation procedure and extraction rate of 9 individual herbs are shown in Table S1) were provided by SPH Xing Ling Sci. \& Tech. Pharmaceutical Co., Ltd. Lansoprazole was procured from Hairong (Dujiangyan, China). Mefenamic acid, D-glucose-6-phosphate (G-6-P), glucose-6phosphate dehydrogenase (G-6-PDH), and $\beta-N_{A D P}{ }^{+}$were supplied by Sigma-Aldrich (St. Louis, MO, USA). Phenacetin, coumarin, paclitaxel, omeprazole, dextromethorphan, chlorzoxazone, testosterone, ketoconazole, and lopinavir were obtained from Meilun Bio. Tech (Dalian, China). Diclofenac was obtained from Ark Pharm (Wuhan, China). 5-Hydroxyomeprazole and $6 \beta$ hydroxytestosterone were purchased from TLC Pharmaceutical Standards (South Aurora, Canada). 6a-Hydroxytaxol and 6hydroxychlorzoxazone were obtained from Torabto Research Chemicals (Ontario, Canada) and 4'-hydroxydiclofenac from Cayman Chemicals (Ann Arbor, Michigan, USA). 7Hydroxycoumarin was supplied by Alfa Aesar (Heysham, UK). The purities of all compounds used in this study were $>98 \%$. $\mathrm{MgCl}_{2}$ and sodium carboxymethyl cellulose (CMC-Na) were obtained from Sinopharm Chemical Reagent (Shanghai, China). Luciferin detection reagent (LDR) was ordered from Promega Biotech (Madison, USA). N-(2-Butyl-1,3-dioxo-2,3-dihydro-1H-phenalen-6-yl)-2-chloroacetamide (NCEN) and its hydrolytic metabolite 4-amino-1,8-naphthalimide (NAH) were synthesized by us according to a previously reported method [30,31]. D-Luciferin methyl ester (DME) and its hydrolytic metabolite D-luciferin were purchased from AAT Bioquest (USA). Licochalcone A, licochalcone
$B$, licochalcone $C$, and licochalcone D were obtained from Baoji Chenguang Biological Technology Co., Ltd. (China). Echinatin was purchased from Shanghai Yuanye Bio-Technology Co., Ltd. (Shanghai, China). Kaempferol, quercetin and rutinum were provided by Chengdu Pufei De Biotech Co., Ltd. (Chengdu, China). Neochlorogenic acid, chlorogenic acid, caffeic acid, isochlorogenic acid $A$, isochlorogenic acid $B$, isochlorogenic acid $C$ and cryptochlorogenic acid were purchased from Shanghai Standard Technology Co., Ltd. (Shanghai, China). Pooled human liver microsomes (Lot No. H0610) from 50 individual donors were supplied by XenoTech (USA). Pooled rat liver microsomes (Lot No. JPXY) were supplied by the Research Institute for Liver Diseases (RILD, Shanghai, China). LC grade methanol, acetonitrile and formic acid were ordered from Fisher Scientific Co., Ltd (Fair Lawn, NJ, USA). Ultra-purified water was prepared using a Millipore purification system.

P450 inhibition assays

Inhibition of human P450s by HEJG, nine herbal extracts, or constituents. P450 inhibition assays were carried out in $200 \mu \mathrm{L}$ reaction mixtures, which included phosphate-buffered saline (PBS, $100 \mathrm{mM}, \mathrm{pH}$ 7.4), each substrate for a target P450, NADPHgenerating system $(10 \mathrm{mM}$ of G-6-P, 1.0 unit/mL of G-6-PDH, 1.0 $\mathrm{mM}$ of $\beta$-NADP ${ }^{+}, 4.0 \mathrm{mM}$ of $\left.\mathrm{MgCl}_{2}\right), \mathrm{HLMs}$ or RLMs, along with HEJG, or each herbal extract or some major constituents in these herbs $[32,33]$. Notably, Herba Schizonepetae oil is a key material for preparing Jingyin granules, while water extracts of other individual herbs were used for preparing Jingyin granules. In this study, Herba Schizonepetae oil was dissolved in acetonitrile; eight other CMs and HEJG were dissolved in ultra-purified water. Each substrate or tested compound was dissolved in acetonitrile, and all solutions were stored at $-20^{\circ} \mathrm{C}$ until use. The final concentration of the organic solvent was $<1 \%(\mathrm{v} / \mathrm{v})$. The probe reactions and experimental details for CYP inhibition assays are listed in Table S2. Briefly, HEJG or its individual herbs $(50-5000 \mu \mathrm{g} / \mathrm{mL}$, final concentrations), together with PBS (pH 7.4), HLMs or RLMs, and the NADPH-generating system were added to the reaction to evaluate inhibitory potentials against P450s. The mixtures were vortexed and then preincubated at $37^{\circ} \mathrm{C}$ for $3 \mathrm{~min}$ or $33 \mathrm{~min}$, and the reactions were initiated by adding individual substrates. The reactions proceeded for $10-30 \mathrm{~min}$ at $37^{\circ} \mathrm{C}$ prior to quenching with $200 \mu \mathrm{L}$ of ice-cold acetonitrile (with internal standard). The protein precipitate was removed by centrifugation at $20,000 \times g$ and $4{ }^{\circ} \mathrm{C}$ for $20 \mathrm{~min}$, and the supernatant $(60 \mu \mathrm{L})$ was mixed with ultrapure water $(60 \mu \mathrm{L})$ at a 1:1 ratio for LC-MS/MS analysis, as described in Table S2 (please refer to the details in the Supplementary Material).

Inactivation kinetic analyses. CYP2C19 and CYP3A inactivation kinetic experiments were performed as previously reported [34-36], with an inactivation group and an activity evaluation group. The final concentration of the organic solvent was $<1 \%$ $(\mathrm{v} / \mathrm{v})$. The inactivation group $(200 \mu \mathrm{L})$ included PBS $(\mathrm{pH} 7.4)$, substrate, the NADPH-generating system, HLMs, and HEJG (at different concentrations). The activity evaluation group $(180 \mu \mathrm{L})$ consisted of PBS ( $\mathrm{pH} 7.4)$, testosterone or omeprazole, the NADPH-generating system, and HLMs. For the inactivation group, the reactions were initiated by adding the NADPHgenerating system after preincubation for $3 \mathrm{~min}$ at $37^{\circ} \mathrm{C}$; the inactivation reaction mixtures $(20 \mu \mathrm{L})$ were transferred to the activity-sensing group at different time points $(0,5,10,15,20$, and $30 \mathrm{~min}$ ). Following incubation for $10-20 \mathrm{~min}$ at $37^{\circ} \mathrm{C}$, icecold acetonitrile $(200 \mu \mathrm{L})$ containing an internal standard was added to quench the oxidative reaction. Activities of CYP2C19 and CYP3A were determined by using the corresponding probe reactions, and metabolites were quantified by LC-MS/MS, as listed in Table S2. The natural logarithm of the residual activity (hydroxylated rates of testosterone and omeprazole) was 
plotted against the preincubation time. All inactivation data were fitted by the following Eq. (1):

$K_{\text {obs }}=K_{\text {inact }} \times I /\left(I+K_{l}\right)$

where $I$ is the final concentration of HEJG; $K_{I}$ is the inactivator (HEJG) concentration at half-maximal inactivation; $K_{\text {inact }}$ is the maximal inactivation rate constant; and $K_{o b s}$ is the observed first-order inactivation rate constant.

\section{CES inhibition assays}

Inhibition of CES1A-catalyzed DME hydrolysis by HEJG. The procedure for hCES1A inhibition assays has been reported previously $[37,38]$ using DME as the probe substrate. Briefly, the $100 \mu \mathrm{L}$ incubation system consisted of PBS (pH 6.5), HLMs, DME and HEJG (at different concentrations). The final concentration of the organic solvent was $<1 \%(\mathrm{v} / \mathrm{v})$. PBS (pH 6.5), HEJG, and HLMs were vortexed and preincubated at $37^{\circ} \mathrm{C}$ for 3 or $33 \mathrm{~min}$, and the reactions were initiated by adding DME and incubated for another $10 \mathrm{~min}$. All reactions were quenched by adding $100 \mu \mathrm{L}$ LDR. A fluorescence microplate reader (SpectraMax ${ }^{\oplus D 3}$, Molecular Devices, Austria) was used to measure the hydrolysis rate of DME by monitoring the rates of formation of the hydrolytic metabolite D-luciferin. The details for the hCES1A inhibition assays and the detection conditions are provided in Table S3.

Inhibition of CES2A-catalyzed NCEN hydrolysis by HEJG. The procedure for hCES2A inhibition assays has also been reported previously [38-40] using NCEN as the probe substrate. Briefly, the $200 \mu \mathrm{L}$ incubation system consisted of PBS (pH 7.4), HLMs, NCEN and HEJG (at different concentrations). The final concentration of the organic solvent was $<1 \%(\mathrm{v} / \mathrm{v})$. PBS (pH 7.4), HEJG, and HLMs were vortexed and preincubated at $37^{\circ} \mathrm{C}$ for 3 or $33 \mathrm{~min}$, and the reactions were initiated by adding NCEN. A fluorescence microplate reader (SpectraMax iD3, Molecular Devices, Austria) was used to measure the hydrolytic rate of NCEN by monitoring the formation rates of the hydrolytic metabolite 4-amino-1,8-naphthalimide (NAH). The details for the hCES2A inhibition assays and the detection conditions for NAH are shown in Table S3.

Pharmacokinetic interactions between HEJG and lopinavir in rats. In this study, all animal tests were approved by the Animal Care and Use Committee of Shanghai Institute of Food and Drug Control with approval No. SIFDC18096. In brief, male SpragueDawley rats $(180-200 \mathrm{~g}, n=6)$ were ordered from Shanghai Laboratory Animal Center (Shanghai, China) and then housed at $25^{\circ} \mathrm{C}$ and a relative humidity of $\sim 55 \%$ in a $12 \mathrm{~h}$ light-dark cycle. Prior to dosing, the rats were fasted overnight with water freely and provided food after finishing the experiment. HEJG was suspended in water, and lopinavir was suspended in $0.5 \%$ CMC$\mathrm{Na}$. According to doctors, the dosage of Jingyin granules can be doubled for severe symptoms in some patients. In this study, the dose of Jingyin granules used in rats was $\sim 6.3$-fold that in humans. HEJG $(3 \mathrm{~g} / \mathrm{kg}, n=3)$ or water $(3 \mathrm{~g} / \mathrm{kg}, n=3)$ was administered intragastrically. After $30 \mathrm{~min}$, lopinavir $(160 \mathrm{mg} / \mathrm{kg}, n=6)$ was administered intragastrically [41-43]. Blood samples were collected at $0,0.25,0.5,0.75,1,2,3,4,6,8,12$, and $24 \mathrm{~h}$, centrifuged for $10 \mathrm{~min}$ at $8000 \mathrm{rpm}$ at $4{ }^{\circ} \mathrm{C}$, and stored at $-80^{\circ} \mathrm{C}$ until analysis. Twenty microlitres of plasma was diluted with acetonitrile (containing internal standard) at a ratio of 1:5 and centrifuged at $20,000 \times g$ at $4^{\circ} \mathrm{C}$ for $30 \mathrm{~min}$. Fifty microlitres of supernatant was diluted with $150 \mu \mathrm{L}$ of Millipore water for LC-MS/MS analysis (Table S4). Quantification of lopinavir $(1-5000 \mathrm{ng} / \mathrm{mL})$ was performed within the linear range of the calibration curve. The pharmacokinetic parameters of lopinavir were fitted by standard noncompartmental analyses using WinNonlin 5.2 (Pharsight Corporation, Mountain View, CA, USA).

Data statistics. Inhibition constants (including $\mathrm{IC}_{50}, K_{l}$, and $k_{\text {inact }}$ values) were calculated by nonlinear regression using GraphPad Prism 6.0 (GraphPad Software, Inc., La Jolla, USA). All data are expressed as the mean \pm SD of triplicate assays.

\section{RESULTS}

Chemical profiling of HEJG by UHPLC-Q-Exactive Orbitrap HRMS First, chemical profiling of HEJG was performed by UHPLC-QExactive Orbitrap HRMS to decipher the major constituents of HEJG. As shown in Fig. S1 and Table S5, a total of 123 constituents were identified from HEJG via comparison with the retention times and MS/MS spectra of the commercially available reference standards or reference with the literature and the MS/MS databases of natural products. These constituents can be classified into several major classes, including 50 flavonoids, 29 organic acids, 10 iridoids, 21 saponins, 5 chalcones, 4 acylamides, and 4 others. Details of the retention times, pseudo-molecular ions and fragment ions of the 123 constituents are listed in Table S5. This results suggest that HEJG contain a variety of natural compounds, comprising a mixture of more than 100 natural ingredients. Alternatively, we assayed the absorbed components in rat plasma following oral administration of HEJG to rats, and 49 prototype chemical constituents were detected. Related information is available in Table S6.

Inhibition of HEJG against major DMEs in HLMs

Next, the inhibition potentials of HEJG against ten major DMEs in HLMs were preliminarily assessed by using three different concentrations $(10,100$, and $1000 \mu \mathrm{g} / \mathrm{mL})$. As shown in Fig. 1, HEJG exhibited concentration-dependent inhibition of all tested DMEs in HLMs. To quantitatively assess the inhibitory effects, concentration-response curves of HEJG against all tested DMEs in

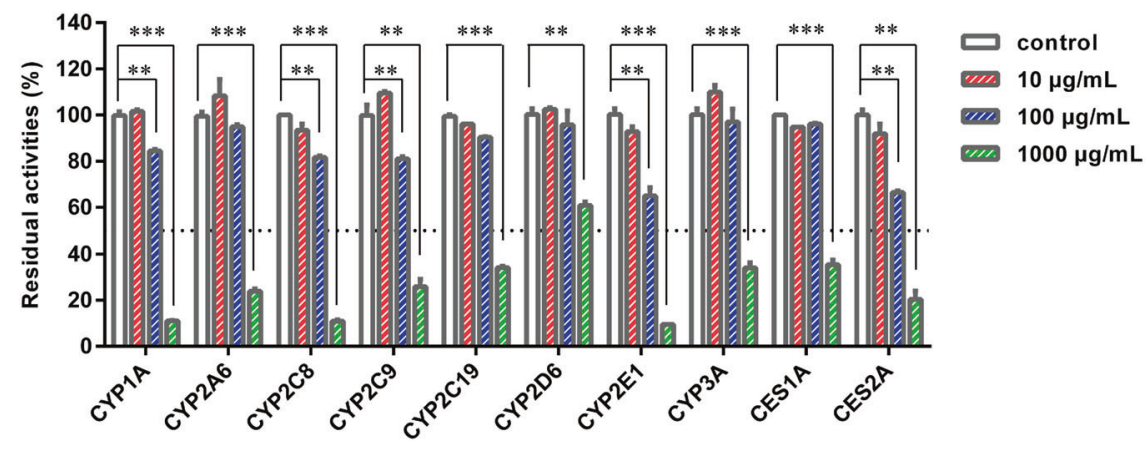

Fig. 1 Inhibition of phase I drug-metabolizing enzymes in HLMs by HEJG. Data are expressed as the mean \pm SD $(n=3)$. ${ }^{* *} P<0.01,{ }^{* * *} P<$ 0.001 compared with the control group. 
Table 1. Inhibitory effects of HEJG on eight major P450s and two CESs in HLMs (with 3 min or with 33 min preincubation).

\begin{tabular}{lllc}
\hline Probe reaction & Target P450 & \multicolumn{2}{l}{ Time-dependent inhibition $\mathrm{IC}_{50}(\mu \mathrm{g} / \mathrm{mL})$} \\
\cline { 3 - 4 } & & Preincubation for 3 min & Preincubation for 33 min \\
\hline Phenacetin O-deethylation & CYP1A & $287.4 \pm 31.5$ & $276.2 \pm 27.8$ \\
Coumarin 7-hydroxylation & CYP2A6 & $750.2 \pm 94.8$ & $664.9 \pm 62.6$ \\
Paclitaxel 6 $\alpha$-hydroxylation & CYP2C8 & $312.6 \pm 2.8$ & $315.2 \pm 16.6$ \\
Diclofenac 4'-hydroxylation & CYP2C9 & $621.4 \pm 55.1$ & $568.0 \pm 47.4$ \\
Omeprazole 5-hydroxylation & CYP2C19 & $614.5 \pm 56.7$ & $272.0 \pm 10.2$ \\
Dextromethorphan O-demethylation & CYP2D6 & $2336.0 \pm 333.2$ & $2484.0 \pm 372.0$ \\
Chlorzoxazone 6-hydroxylation & CYP2E1 & $810.0 \pm 154.4$ & $868.8 \pm 123.4$ \\
Testosterone 6 $\beta$-hydroxylation & CYP3A & $756.9 \pm 61.3$ & $191.3 \pm 7.7$ \\
DME hydrolysis & hCES1A & $717.5 \pm 83.6$ & $987.5 \pm 123.2$ \\
NCEN hydrolysis & hCES2A & $219.0 \pm 19.9$ & 0.99 \\
\hline
\end{tabular}
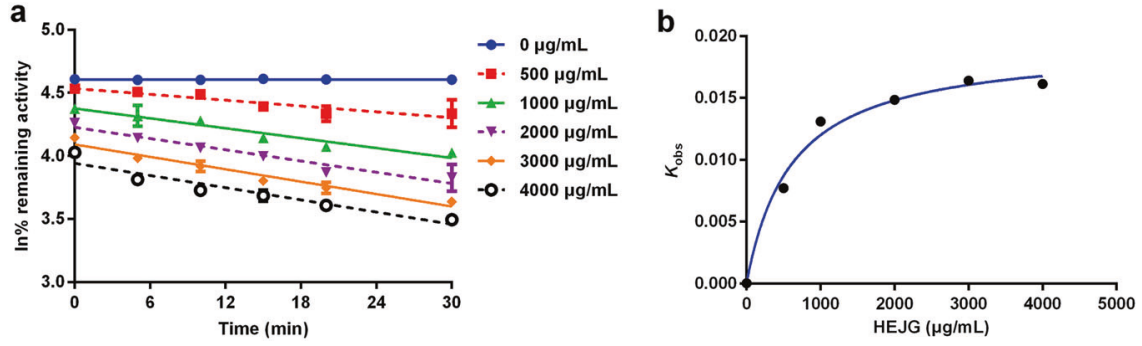

Fig. 2 Time-dependent inhibition of CYP3A in HLMs by HEJG. a Time- and dose-dependent inhibition of CYP3A by HEJG. $\mathbf{b}$ The hyperbolic plot of $K_{o b s}$ of CYP3A vs. HEJG concentrations. Data are expressed as the mean \pm SD $(n=3)$.

HLMs were plotted. As shown in Figs. S2, S3 and Table 1, HEJG concentration-dependently inhibited the catalytic activities of CES1A, CES2A, CYPs1A, 2A6, 2C8, 2C9, 2C19, 2D6, 2E1, and 3 A, with calculated $I_{50}$ values of $717.5,219.0,287.4,750.2,312.6$, $621.4,614.5,2336.0,810.0$, and $756.9 \mu \mathrm{g} / \mathrm{mL}$, respectively.

To determine whether HEJG inhibited these human DMEs in a time-dependent manner, the residual enzymatic activities of ten DMEs following different preincubation periods were investigated in HLMs in the presence of increasing concentrations of HEJG. As illustrated in Figs. S2, S3 and Table 1, following a long (33 min) preincubation of HEJG with HLMs at $37^{\circ} \mathrm{C}$, HEJG concentrationdependently inhibited the catalytic activities of CES1A, CES2A, CYP1A, CYP2A6, CYP2C8, CYP2C9, CYP2C19, CYP2D6, CYP2E1, and CYP3A, with $\mathrm{IC}_{50}$ values of 987.5, 203.8, 276.2, 664.9, 315.2, 568.0, $272.0,2484.0,868.8$, and $191.3 \mu \mathrm{g} / \mathrm{mL}$, respectively. Notably, a long preincubation time resulted in much more potent inhibitory effects of HEJG against both CYP2C19 (the $\mathrm{IC}_{50}$ value decreased from 614.5 to $272.0 \mu \mathrm{g} / \mathrm{mL}$ ) and CYP3A (the $\mathrm{IC}_{50}$ value decreased from 756.9 to $191.3 \mu \mathrm{g} / \mathrm{mL}$ ), with changes in $I_{50}$ value of $>2.0$ fold. These findings suggest that HEJG contains naturally occurring inactivators of human CYP2C19 and CYP3A, which might inactivate CYP2C19 and CYP3A (mainly) in vivo and result in undesirable effects, such as time-dependent inhibition of these two P450 enzymes or idiosyncratic toxicity.

Inactivation kinetics of HEJG against CYP2C19 and CYP3A in HLMs To further characterize the time-dependent inhibition of HEJG against human CYP2C19 and CYP3A, the inactivation kinetics of HEJG against CYP2C19 and CYP3A in HLMs were evaluated; kinetic parameters (including $K_{l}$ and $k_{\text {inact }}$ values) were determined according to a previously reported procedure. In the presence of NADPH, HEJG inactivated CYP2C19 and CYP3A in concentrationand time-dependent manners (Figs. S4, Fig. 2). As calculated from the plots presented in Figs. S4, Fig. 2, the inactivation kinetic constants of HEJG against CYP2C19, including $K_{l}$ and $K_{\text {inact }}$, were determined to be $1038.0 \mu \mathrm{g} / \mathrm{mL}$ and $0.018 \mathrm{~min}^{-1}$, respectively; those of HEJG against CYP3A were $612.9 \mu \mathrm{g} / \mathrm{mL}$ and $0.019 \mathrm{~min}^{-1}$, respectively. These results clearly demonstrate that HEJG inactivates CYP2C19 and CYP3A in concentration-, NADPH-, and timedependent manners, suggesting that Jingyin granules might result in undesirable effects via inactivation of CYP2C19 and CYP3A.

Inhibition of HEJG against Cyp2c and Cyp3a in RLMs Next, the inhibitory effects of HEJG against Cyp2c and Сyp3a in RLMs were investigated following different preincubation periods ( 3 or $33 \mathrm{~min}$ ) at $37^{\circ} \mathrm{C}$. As depicted in Fig. S5, a long preincubation (33 min) of HEJG with RLMs resulted in significant loss of rat Cyp3a activity; the $\mathrm{IC}_{50}$ value decreased from 944.8 to $350.0 \mu \mathrm{g} / \mathrm{mL}$, with an $\mathrm{IC}_{50}$ ratio of 2.70 -fold. In sharp contrast, the $\mathrm{IC}_{50}$ value of HEJG against rat Cyp2c increased from 594.3 to $991.3 \mu \mathrm{g} / \mathrm{mL}$, with an $\mathrm{IC}_{50}$ ratio of 0.60 -fold. These findings clearly suggest that HEJG inhibits rat Сур3a in a time-dependent manner but does not trigger this inactivation effect of rat Cyp2c (Fig. 2). Thus, although it is unfeasible to test the in vivo effects of HEJG against CYP2C, it is feasible and meaningful to study pharmacokinetic interactions between HEJG and CYP3A-substrate drugs in vivo, with rats used as a model for humans.

Pharmacokinetic interactions between HEJG and lopinavir in rats On the basis of the above-mentioned findings, the influence of HEJG on the pharmacokinetic behavior of CYP3A substrate-drug(s) was investigated in vivo in rats. As shown in Fig. 3 and Table 2, following co-administration of HEJG and lopinavir, the elimination half-life $\left(t_{1 / 2}\right)$ of lopinavir in rats was prolonged by 1.91 -fold (from 1.40 to $2.68 \mathrm{~h}$ ); the $A U C_{0 \text {-inf }}$ of lopinavir in rats was significantly increased by 2.43 -fold (from 4189 to $10196 \mathrm{ng} / \mathrm{mL} \mathrm{h}$ ). In addition, the $C_{\max }$ value of lopinavir in rat plasma increased slightly from 


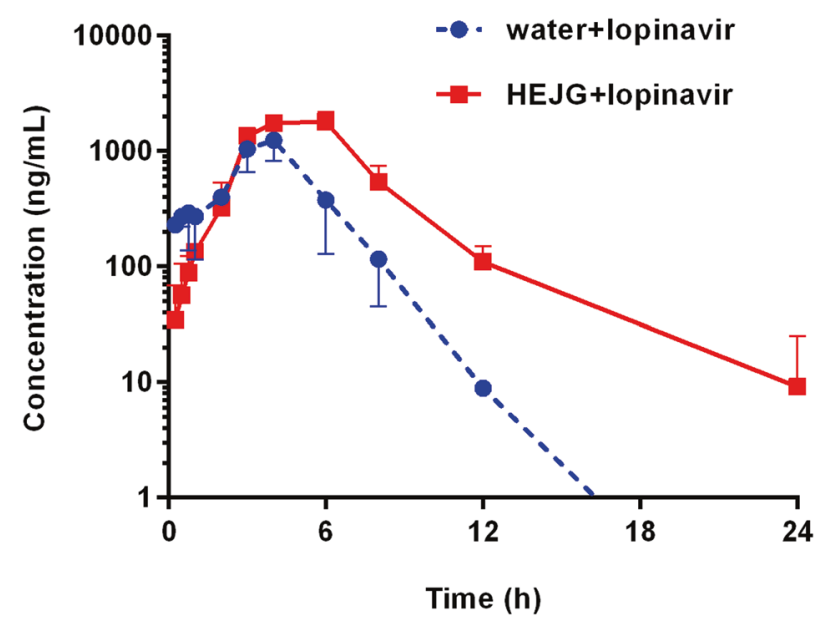

Fig. 3 Mean plasma concentration-time curves of lopinavir (160 $\mathrm{mg} / \mathrm{kg}$, i.g.) in the control group (water+lopinavir, $n=3$ ) and experimental group (HEJG+lopinavir, $n=3$ ).

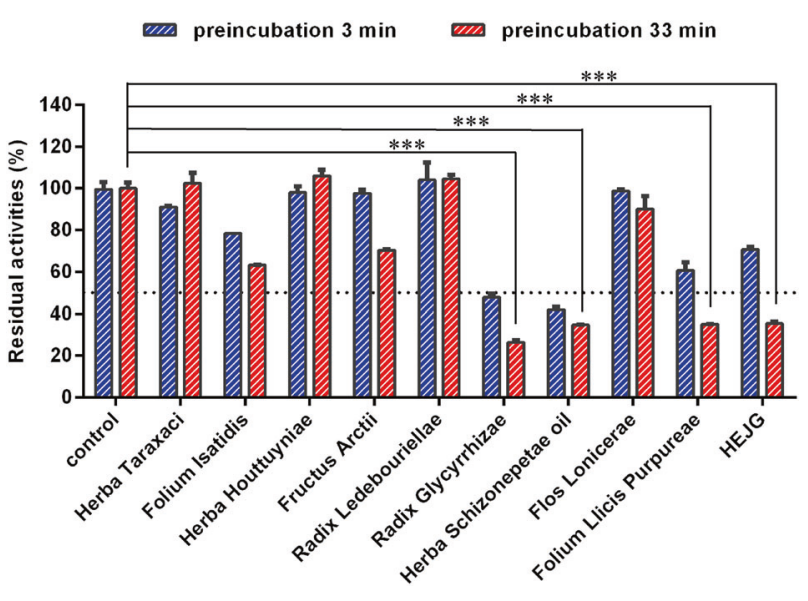

Fig. 4 Inhibitory potentials of individual extracts of nine different herbs used for preparing Jingyin granules $(250 \mu \mathrm{g} / \mathrm{mL})$ against CYP3A-catalyzed testosterone $\mathbf{6} \boldsymbol{\beta}$-hydroxylation in HLMs. Data are expressed as the mean $\pm \mathrm{SD}(n=3)$. ${ }^{* * *} P<0.001$ compared with the control group with 33 min of preincubation.

Table 2. The impact of HEJG on the pharmacokinetic parameters of lopinavir in rats.

\begin{tabular}{llll}
\hline & $\begin{array}{l}\text { AUC } \\
(\mathrm{ng} / \mathrm{mL} \cdot \mathrm{h})\end{array}$ & $C_{\max }(\mathrm{ng} / \mathrm{mL})$ & $t_{1 / 2}(\mathrm{~h})$ \\
\hline Water + lopinavir & $4189 \pm 131$ & $1275 \pm 55$ & $1.40 \pm 0.23$ \\
HEJG + lopinavir & $10196 \pm 1574$ & $1855 \pm 215$ & $2.68 \pm 0.45$ \\
Ratio & 2.43 & 1.45 & 1.91 \\
\hline Mean \pm SD of triplicate rats. & & & $6.00 \pm 0.33$ \\
\hline
\end{tabular}

\begin{tabular}{|c|c|c|c|c|}
\hline Herbs & Extraction rate (\%) & Preincubation for $3 \mathrm{~min}$ & Preincubation for $33 \mathrm{~min}$ & Ratio \\
\hline Radix Glycyrrhizae & 31.1 & $211.2 \pm 19.9$ & $101.8 \pm 7.8$ & 2.07 \\
\hline Folium Llicis Purpureae & 29.4 & $419.8 \pm 42.0$ & $209.2 \pm 8.1$ & 2.01 \\
\hline Herba Schizonepetae oil & $0.005952 \mathrm{~mL} / \mathrm{g}$ & $267.7 \pm 19.7$ & $346.6 \pm 57.8$ & 0.77 \\
\hline
\end{tabular}

1275 to $1855 \mathrm{ng} / \mathrm{mL}$. It is evident from these findings that Jingyin granules can strongly modulate the pharmacokinetic profiles of lopinavir in rats by increasing plasma exposure of lopinavir and prolonging the plasma half-life of lopinavir in rats.

Inactivation effects of CYP3A by the individual herbs for preparing HEJG

Subsequently, time-dependent inhibition of CYP3A by individual extracts of nine different herbs for preparing HEJG $(250 \mu \mathrm{g} / \mathrm{mL}$, final concentration) was conducted to determine the key herbs in Jingyin granules that cause CYP3A inactivation. As shown in Fig. 4, Radix Glycyrrhizae (Gancao), Folium Llicis Purpureae (Sijiqing) and Herba Schizonepetae oil (Jingjie) displayed relatively strong CYP3A inhibition activities, as residual CYP3A activities were $<50 \%$ at a dose of $250 \mu \mathrm{g} / \mathrm{mL}$. Then, the inhibition and inactivation effects of these three individual herbs for CYP3A were carefully investigated in HLMs. As illustrated in Fig. S6 and Table 3, Radix Glycyrrhizae (Gancao) and Folium Llicis Purpureae (Sijiqing) inhibited CYP3Acatalyzed $6 \beta$-hydroxylation of testosterone in HLMs in a time- and $\mathrm{NADPH}$-dependent manner, with $\mathrm{IC}_{50}$ ratios of 2.07-fold and 2.01- fold, respectively. These findings clearly demonstrate that Radix Glycyrrhizae (Gancao) and Folium Llicis Purpureae (Sijiqing) are the key herbs in Jingyin granules, resulting in significant loss of CYP3A activity in the NADPH-generating system.

Inactivation effects of the constituents in Gancao and Sijiqing on CYP3A

Next, inactivation effects of the constituents isolated from Radix Glycyrrhizae (Gancao) and Folium Llicis Purpureae (Sijiqing) on CYP3A were carefully investigated. As the chalcones in Radix Glycyrrhizae (Gancao) are a class of compounds bearing Michael acceptor(s), their inactivation effects against CYP3A were studied first. As shown in Fig. S7 and Table 4, licochalcone A, licochalcone $\mathrm{B}$, licochalcone $\mathrm{C}$ and echinatin in Radix Glycyrrhizae inhibited CYP3A-catalyzed testosterone $6 \beta$-hydroxylation in a timedependent manner, with $\mathrm{IC}_{50}$ ratios of 2.15 -fold, 2.07-fold, 2.40fold, and 3.20-fold, respectively. Second, the major constituents in Folium Llicis Purpureae (Sijiqing) were identified by UHPLC-QExactive Orbitrap HRMS, and inactivation effects of the commercially available constituents against CYP3A were investigated. As 
Table 4. The inhibitory effects of the chalcones in Radix Glycyrrhizae (Gancao) and the major constituents in Folium Llicis Purpureae (Sijiqing) on CYP3A in HLMs (with 3 min or with 33 min preincubation).

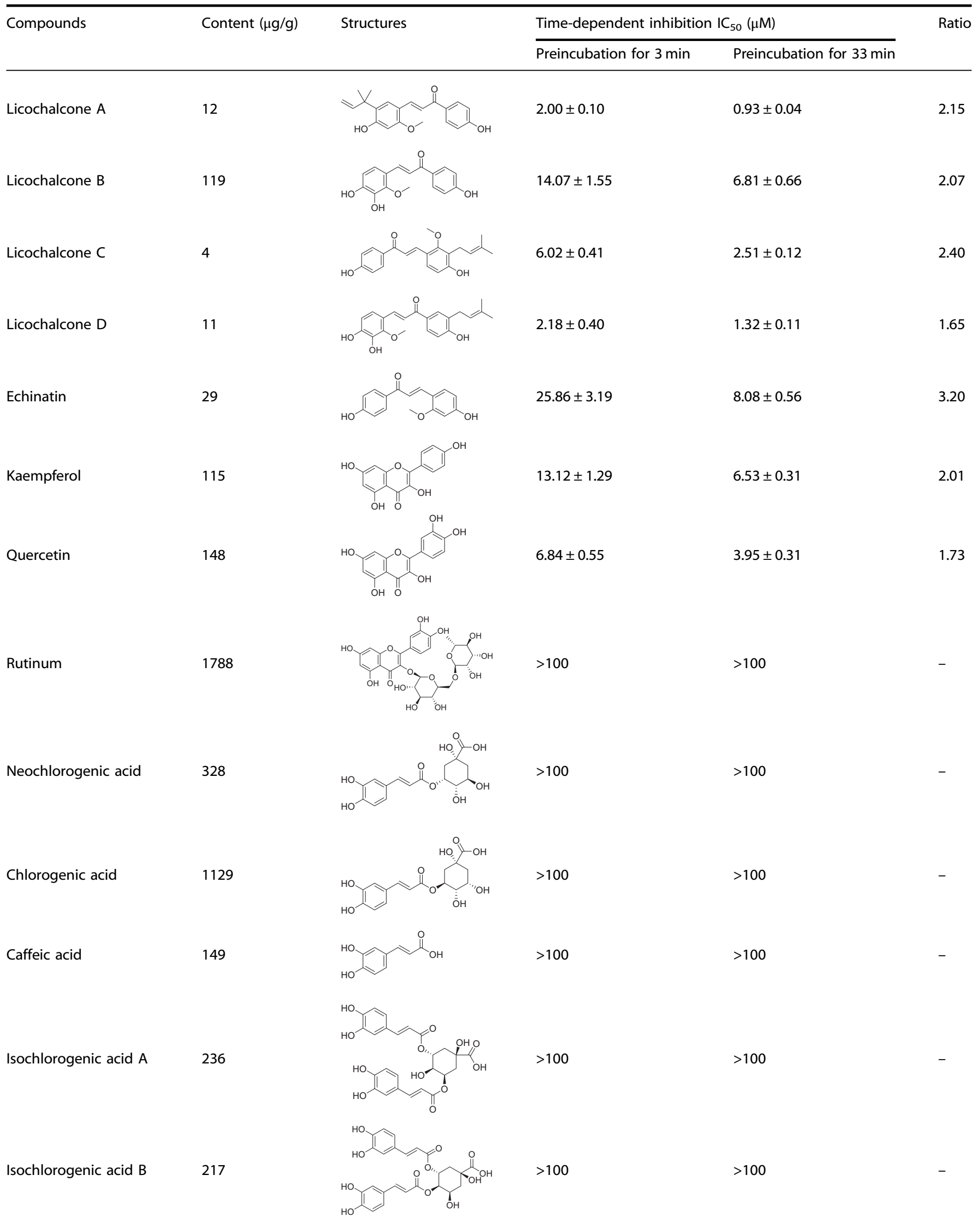


Table 4. continued

\begin{tabular}{|c|c|c|c|c|c|}
\hline \multirow[t]{2}{*}{ Compounds } & \multirow[t]{2}{*}{ Content $(\mu \mathrm{g} / \mathrm{g})$} & \multirow[t]{2}{*}{ Structures } & \multicolumn{2}{|c|}{ Time-dependent inhibition $\mathrm{IC}_{50}(\mu \mathrm{M})$} & \multirow[t]{2}{*}{ Ratio } \\
\hline & & & Preincubation for $3 \mathrm{~min}$ & Preincubation for $33 \mathrm{~min}$ & \\
\hline Isochlorogenic acid C & 348 & & $>100$ & $>100$ & - \\
\hline Cryptochlorogenic acid & 360 & & $>100$ & $>100$ & - \\
\hline
\end{tabular}

Data are expressed as mean \pm SD $(n=3)$.

depicted in Figs. S7, S8 and Table 4, kaempferol and quercetin in Folium Llicis Purpureae (Sijiqing) inhibited CYP3A-catalyzed $6 \beta$ hydroxylation of testosterone in a time-dependent manner; the $\mathrm{IC}_{50}$ ratios were 2.01 -fold and 1.73 -fold, respectively. Furthermore, neochlorogenic acid, chlorogenic acid, caffeic acid, isochlorogenic acid $A$, isochlorogenic acid $B$, isochlorogenic acid $C$, and cryptochlorogenic acid $(100 \mu \mathrm{M})$, compounds bearing Michael acceptor(s), showed weak inhibitory effects against CYP3A, and the inhibition was slightly time dependent. These findings demonstrate that licochalcone $A$, licochalcone $B$, licochalcone $C$ and echinatin in Radix Glycyrrhizae, as well as kaempferol and quercetin in Folium Llicis Purpureae, inhibit CYP3A-catalyzed testosterone $6 \beta$-hydroxylation in a time-dependent manner.

\section{DISCUSSION}

Accompanied by the widespread occurrence of influenza and COVID-19, a variety of Western therapeutics and herbal medicines have been approved or recommended for the treatment or adjuvant treatment of viral diseases in clinical settings [44]. Over the past 10 months, more than ten herbal medicines or Chinese medicine compound formulas (such as Qingfei Paidu decoction, Jingyin granules, Banlangen granules and Lianhua Qingwen capsules) have been recommended by the National Health Commission of the People's Republic of China or the local governments in China for combating the COVID-19 epidemic. As the only herbal medicine recommended for preventing and treating COVID-19 by the Shanghai municipal government, Jingyin granules have been validated to play an active role in combating this epidemic, especially in alleviating the moderate and mild symptoms (such as fever, muscle pain and imaging manifestations) of some patients. In clinical settings, Jingyin granules have been frequently used in combination with a range of Western therapeutic agents (such as antiviral drugs, anti-inflammatory drugs, immunosuppressive agents, and other Western medicines) for combating COVID-19 and other viral epidemics. However, use of Jingyin granules together with Western agents may alter the outcomes of the latter, leading to decreased or increased effectiveness or adverse effects. Thus, it is urgent and necessary to carefully assess potential HDls between Jingyin granules and co-administered Western therapeutics.

It is well known that pharmacokinetic interactions are the major cause of clinically relevant $\mathrm{HDIs}[25,44,45]$, whereby herbal medicines modulate the pharmacokinetic behavior of coadministered Western drug(s) by regulating the expression or function of DMEs and transporters responsible for the metabolic elimination of victim Western drug(s). Because most marketed
Western antiviral drugs (Table S7, such as oseltamivir, remdesivir, and lopinavir) are predominantly metabolized by phase I DMEs, including P450s and CESs [27-29], this study focused on assessing potential HDIs between HEJG and antiviral Western drugs via inhibition or inactivation of phase I DMEs. The results clearly demonstrate that HEJG dose-independently inhibited all tested human DMEs, including human CES1A, CES2A, CYPs1A, 2A6, 2C8, $2 \mathrm{C} 9,2 \mathrm{C} 19,2 \mathrm{D} 6,2 \mathrm{E} 1$, and $3 \mathrm{~A}$, with $\mathrm{IC}_{50}$ values ranging from 219.0 to $2336.0 \mu \mathrm{g} / \mathrm{mL}$. Notably, this herbal medicine time- and NADPHdependently inhibited human CYP2C19 and CYP3A, two key enzymes participating in the metabolism of a wide range of Western drugs, including some marketed antiviral agents (such as lopinavir and nelfinavir) and some agents with very narrow therapeutic windows (such as digoxin and warfarin). Overall, increasing evidence suggests that time-dependent inhibition of CYPs may lead to serious drug/herbal-drug interactions and druginduced idiosyncratic toxicity, even though the in vivo effects of reversible CYP inhibitors are relatively minor [46, 47]. These findings encourage us to further investigate the influence of HEJG on the pharmacokinetic behavior of CYP2C19- or CYP3A-substrate agents in vivo.

Prior to in vivo testing, we investigated species differences in CYP inactivation by HEJG, and the results showed that HEJG inhibited both human CYP3A and rat Cyp3a in dose-, time-, and NADPH-dependent manners but it inhibited rat Cyp2c in a reversible manner in RLMs. Hence, it was feasible and meaningful to investigate the pharmacokinetic interactions between HEJG and CYP3A-substrate drugs in vivo using rats as a model, but it was meaningless to investigate the impact of HEJG on the pharmacokinetic behavior of CYP2C19 substrate drugs in rats. Lopinavir (a CYP3A4-substrate drug), a commonly used antiviral agent for treating severe acute respiratory syndrome and COVID19, was selected as the victim drug for studying in vivo pharmacokinetic interactions between Jingyin granules and CYP3A4-substrate drug(s). The results clearly showed that HEJG significantly prolonged the elimination half-life of lopinavir by 1.91 -fold and strongly elevated the plasma exposure of this antiviral agent by $2.43-$ fold when HEJG $(3 \mathrm{~g} / \mathrm{kg}$ ) was administered with lopinavir $(160 \mathrm{mg} / \mathrm{kg})$ in rats. These findings demonstrate that HEJG inhibits CYP3A both in vitro and in vivo, suggesting that much attention should be given when Jingyin granules are coadministered with CYP3A-substrate drugs, particularly agents with narrow therapeutic windows (such as digoxin and warfarin, which are predominantly metabolized by CYP3A). Both intestinal and hepatic CYP3A participate in lopinavir-related drug interactions, and considering that the plasma exposure of the absorbed components following oral administration of HEJG is very low, we speculate that HEJG-mediated HDI may occur mainly through 
inhibition of intestinal CYP3A. A single dose of HEJG in humans is $5.2 \mathrm{~g}$ (herbal extract). Based on US FDA drug-drug interaction study guidance (2017), the human intestinal luminal concentration of HEJG was calculated to be $20.8 \mathrm{~g} / \mathrm{L}$. Notably, HEJG dosedependently inhibited the catalytic activities of CYP3A, with a calculated $I C_{50}$ value of $756.9 \mu \mathrm{g} / \mathrm{mL}$, and the inactivation kinetic constant $\left(K_{l}\right)$ of HEJG against CYP3A was determined to be 612.9 $\mu \mathrm{g} / \mathrm{mL}$. It is evident that local exposure to HEJG is much higher than the $I C_{50}$ value of HEJG against CYP3A. Thus, clinicians and patients should use the combination of Jingyin granules and antiviral agent(s) carefully because this herbal medicine can strongly inhibit intestinal CYP3A. Similarly, Ravi et al. found that plasma levels of lopinavir increased significantly upon coadministration with grapefruit juice (a CYP3A inhibitor) following single-dose or multidose administration [48], an interaction that was mainly mediated by intestinal CYP3A. It is conceivable that inhibition of intestinal CYP3A by HEJG may also increase the plasma levels of lopinavir in vivo, which can partially explain why HEJG significantly increased the lopinavir plasma exposure by 2.43-fold.

It should be noted that inhibition of human CYPs is always a double-edged sword. For drugs with a sufficiently wide therapeutic window, such as lopinavir (an antiviral drug that is metabolized by CYP3A4), the in vivo efficacy may be enhanced because this agent displays relatively high safety profiles $[49,50]$. Considering that lopinavir is frequently used for antiviral therapy in combination with other antiviral drugs or herbal medicines, the daily dose of lopinavir can be partially reduced to avoid potential DDIs/HDIs when it is co-administered with other antiviral drugs or herbal medicines in consecutive dosages. Notably, COVID-19 is a complex systemic disease, and severe COVID-19 patients often have excessive inflammation and abnormal coagulation [51-56]. To successfully treat severe COVID-19, the combination of antiviral agents, anti-inflammatory drugs and anti-coagulant agents (such as warfarin, a common anti-coagulant agent but with a very narrow therapeutic window) is frequently used. On the one hand, CYP1A and CYP3A play crucial roles in the formation of some key inflammatory factors (such as the oxidative metabolites of arachidonic acid), and strong inhibition of CYP1A and CYP3A may be helpful for the anti-inflammatory effects of Jingyin granules [57-59]. On the other hand, the in vivo anti-coagulant effects of warfarin should be carefully monitored. Although it is unfeasible to investigate the in vivo effects of HEJG against CYP2C19 by using rats as a surrogate model to replace humans, it is conceivable that co-administration of Jingyin granules with warfarin to patients may trigger severe drug interactions because this herbal medicine can strongly inhibit both CYP2C19 and CYP3A in HLMs in a time-dependent manner. Thus, monitoring should be carried out when Jingyin granules are co-administered with this anti-coagulant agent.

Considering that Jingyin granules are prepared from nine herbs that contain hundreds of chemical constituents (refer to Fig. S1 and Table S5), it is unfeasible to identify which ingredients in Jingyin granules are responsible for CYP3A inactivation. Hence, we assayed the inhibitory effects of the extract of each herb on human CYP3A. The results clearly demonstrated that the extract of three herbs used for preparing Jingyin granules strongly inhibit CYP3A-catalyzed 6 $\beta$-hydroxylation of testosterone in a dose-dependent manner. Among them, Herba Schizonepetae oil (Jingjie) inhibited CYP3A-catalyzed testosterone $6 \beta$-hydroxylation in a reversible manner, and Radix Glycyrrhizae (Gancao) and Folium Llicis Purpureae (Sijiqing) inhibited CYP3A-catalyzed testosterone $6 \beta$-hydroxylation in time-, dose- and NADPH-dependent manners. It has been reported that licochalcone $A$ and glabridin isolated from Radix Glycyrrhizae are potent time-dependent inhibitors of CYP3A $[60,61]$, but the key ingredients from Folium Llicis Purpureae (Sijiqing) responsible for time-dependent inhibition of CYP3A have not been reported. Additionally, licochalcone A, licochalcone B, echinatin and licochalcone C in Radix Glycyrrhizae inactivated CYP3A in a dose-dependent manner; kaempferol and quercetin in Folium Llicis Purpureae (Sijiqing) were also identified as CYP3A time-dependent inhibitors. Furthermore, neochlorogenic acid, chlorogenic acid, caffeic acid, isochlorogenic acid $A$, isochlorogenic acid $B$, isochlorogenic acid $C$ and cryptochlorogenic acid $(100 \mu \mathrm{M})$, compounds bearing Michael acceptor(s), showed weak inhibitory effects against CYP3A, and the inhibition was slightly time dependent. However, there are several glycosides of kaempferol and quercetin in HEJG. Reynoutrin, hyperoside, rutinum, and avicularin are glycosides of quercetin, and afzeloside, kaempferol 3-rutinoside, and astragalin are glycosides of kaempferol. Notably, rutinum and astragalin are the major constituents of Folium Llicis Purpureae (Sijiqing). These glycosides can be readily converted to quercetin or kaempferol in the intestinal tract, in turn inactivating intestinal CYP3A and triggering potential HEJG-drug interactions. All the findings presented in this study will be very helpful for guiding the optimization of this herbal prescription to reduce the potential risks of HDIs through CYP3A inactivation. In the future, more in-depth investigation should be performed to investigate whether use of Jingyin granules together with CYP3A-substrate drugs (including but not limited to antiviral agents) triggers clinically relevant drug interactions or other undesirable side effects in vivo.

\section{CONCLUSION}

In summary, this study investigated the inhibitory potency and inhibitory kinetics of the HEJG against human phase I DMEs and assessed potential HDls between Jingyin granules and Coadministered CYP substrate-drug(s) in vivo. The results clearly demonstrated that HEJG dose-dependently inhibited all tested human DMEs in HLMs; inhibition of CYP2C19 and CYP3A occurred in time- and NADPH-dependent manners. Further investigation showed that HEJG also inhibited rat Cyp3a in timeand NADPH-dependent manners but did not trigger inactivation of rat Cyp2c, suggesting that it was feasible and meaningful to study in vivo pharmacokinetic interactions between HEJG and CYP3A-substrate drugs by using rats as a model for humans. In vivo assays revealed that the elimination half-life $\left(t_{1 / 2}\right)$ of lopinavir was be prolonged by 1.91 -fold; the $A U C_{0 \text {-inf }}$ of lopinavir was significantly increased by 2.43 -fold following coadministration of HEJG and lopinavir in rats. Furthermore, time-dependent inhibition assays of CYP3A by the extract of nine individual herbs for preparing Jingyin granules suggested that Radix Glycyrrhizae (Gancao) and Folium Llicis Purpureae (Sijiqing) are responsible for CYP3A inactivation. Further analysis demonstrated that licochalcone $A$, licochalcone $B$, licochalcone $C$, echinatin, kaempferol and quercetin inhibit CYP3A-catalyzed testosterone $6 \beta$-hydroxylation in a time-dependent manner. Collectively, our findings reveal that HEJG significantly modulate the pharmacokinetics of CYP substrate-drug(s) via inactivation of CYP3A, offering key information for patients and clinicians to avoid potential HDIs in antiviral therapy.

\section{ACKNOWLEDGEMENTS}

This work was supported by Shanghai Science and Technology Innovation Action Plans (20S21901500, 20S21900900) supported by Shanghai Science and Technology Committee, the NSF of China (81922070, 81973286), the National Key Research and Development Program of China (2017YFC1700200, 2017YFC1702000), the National Science and Technology Major Project of China (2018ZX09731016), the Three-year Action Plan of Shanghai TCM Development (ZY-(2018-2020)-CCCX-5001), Program of Shanghai Academic/Technology Research Leader (18XD1403600), Shuguang Program (18SG40) \& the Project on the Prevention and Treatment of COVID-19 with Chinese and Western Medicines supported by Shanghai Education Development Foundation and Shanghai Municipal Education Commission. 


\section{AUTHOR CONTRIBUTIONS}

GBG and $\mathrm{HZC}$ designed the study. $\mathrm{FZ}, \mathrm{WL}$, and JH performed the experiments. GBG, QLC, DDW, LWZ, and YFZ analyzed the data. GBG and FZ wrote the paper. WDZ, HZC, and JGX critically revised the paper.

\section{ADDITIONAL INFORMATION}

Supplementary information The online version contains supplementary material available at https://doi.org/10.1038/s41401-021-00697-2.

Competing interests: The authors declare no competing interests.

\section{REFERENCES}

1. Zitzmann C, Kaderali L. Mathematical analysis of viral replication dynamics and antiviral treatment strategies: from basic models to age-based multi-scale modeling. Front Microbiol. 2018;9:1546.

2. Boretti A, Banik B, Castelletto S. Use of ultraviolet blood irradiation against viral infections. Clin Rev Allergy Immunol. 2020;7:1-12.

3. Shah VK, Firmal P, Alam A, Ganguly D, Chattopadhyay S. Overview of immune response during SARS-CoV-2 infection: lessons from the past. Front Immunol. 2020;11:1949.

4. Hoang BX, Shaw G, Fang W, Han B. Possible application of high-dose vitamin C in the prevention and therapy of coronavirus infection. J Glob Antimicrob Resist. 2020;23:256-62.

5. Jang S, Rhee JY. Three cases of treatment with nafamostat in elderly patients with COVID-19 pneumonia who need oxygen therapy. Int J Infect Dis. 2020;96:500-2.

6. Rizk JG, Kalantar-Zadeh K, Mehra MR, Lavie CJ, Rizk Y, Forthal DN. Pharmacoimmunomodulatory therapy in COVID-19. Drugs. 2020;80:1267-92.

7. Gao K, Song YP, Chen H, Zhao LT, Ma L. Therapeutic efficacy of Qingfei Paidu decoction combined with antiviral drugs in the treatment of corona virus disease 2019: a protocol for systematic review and meta analysis. Medicine. 2020;99: e20489.

8. Wang L, Xu X, Ruan J, Lin S, Jiang J, Ye H. Quadruple therapy for asymptomatic COVID-19 infection patients. Expert Rev Anti Infect Ther. 2020;18:617-24.

9. Xin S, Cheng X, Zhu B, Liao X, Yang F, Song L, et al. Clinical retrospective study on the efficacy of Qingfei Paidu decoction combined with Western medicine for COVID-19 treatment. Biomed Pharmacother. 2020;129:110500.

10. Wu L, Chen Y, Ma Y, Yang Z, Yang N, Deng W, et al. Clinical practice guideline on treating influenza in adult patients with Chinese patent medicines. Pharmacol Res. 2020;160:105101.

11. Yu T, Liu XJ, Liu L, Gao Q. Research on the mechanism of Jingyin Granules in the treatment of viral influenza based on network pharmacology. Chin Pat Med. 2020;42:456-61.

12. Zhuang W, Fan $Z$, Chu $Y$, Wang $H$, Yang $Y$, Wu L, et al. Chinese patent medicines in the treatment of coronavirus disease 2019 (COVID-19) in China. Front Pharmacol. 2020;11:1066.

13. Li KY, An W, Xia F, Chen M, Yang P, Liao YL, et al. Retrospective review of Qingfei Paidu Decoction combined with antiviral drugs in the treatment of new coronavirus pneumonia research. Chin Herb Med. 2020;51:2046-9.

14. Yang C, Wang Y, He J, Yan W, Jiang H, Chen Q, et al. Lianhua-Qingwen displays antiviral and anti-inflammatory activity and synergistic effects with oseltamivir against influenza B virus infection in the mouse model. Evid Based Complement Alternat Med. 2020;2020:3196375.

15. Shuguang Hospital Affiliated to Shanghai University of Traditional Chinese Medicine successfully independently developed the first new Chinese medicine drug. J Shanghai Univ Tradit Chin Med. 2009;23:76.

16. Wang YL, Zhang CC, Zhang F, Huang J, Zhao YF, Liu W, et al. UHPLC-Q-Exactive Orbitrap HR-MS analysis of chemical components and tissue distribution characteristics of Jingyin granules. Chin J Chin Mat Med. 2020;45:5537-54.

17. He T, Tang Q, Zeng N, Gou L, Liu JW, Yang J, et al. Study on the anti-influenza virus effect and mechanism of the volatile oil of Nepeta sinensis and its main components. Chin J Chin Mat Med. 2013;38:1772-7.

18. Zhou W, Yin A, Shan J, Wang S, Cai B, Di L. Study on the rationality for antiviral activity of flos lonicerae japonicae-fructus forsythiae herb chito-oligosaccharide via integral pharmacokinetics. Molecules. 2017;22:654

19. Li S, Wang R, Zhang Y, Zhang X, Layon AJ, Li Y, et al. Symptom combinations associated with outcome and therapeutic effects in a cohort of cases with SARS. Am J Chin Med. 2006;34:937-47.

20. Deng YP, Liu YY, Liu Z, Li J, Zhao LM, Xiao H, et al. Antiviral activity of Folium isatidis derived extracts in vitro and in vivo. Am J Chin Med. 2013;41:957-69.

21. Sohn S, Bang D, Lee SI, Kim YA, Lee ES, Ha JY, et al. Combined treatment with colchicine and Herba Taraxaci (Tarazacum mongolicum Hand.-Mazz.) attenuates
Behcet's disease-like symptoms in mice and influences the expressions of cytokines. Int Immunopharmacol. 2003;3:713-21.

22. Kobayashi M, Davis SM, Utsunomiya T, Pollard RB, Suzuki F. Antiviral effect of gingyo-san, a traditional Chinese herbal medicine, on influenza A2 virus infection in mice. Am J Chin Med. 1999;27:53-62.

23. Li H, Liu L. Experimental study on antibacterial and antiviral effects of Fangfengjiiere mixture. Chin Med Mater. 2020;10:2538-43.

24. Yue GG, Chan BC, Kwok HF, To MH, Hon KL, Fung KP, et al. Screening for antiinflammatory and bronchorelaxant activities of 12 commonly used Chinese herbal medicines. Phytother Res. 2012;26:915-25.

25. Ge GB. Deciphering the metabolic fates of herbal constituents and the interactions of herbs with human metabolic system. Chin J Nat Med. 2019;17:801-2

26. Zhou QH, Zhu YD, Zhang F, Song YQ, Jia SN, Zhu L, et al. Interactions of drugmetabolizing enzymes with the Chinese herb Psoraleae Fructus. Chin J Nat Med. 2019;17:858-70.

27. Warren TK, Jordan R, Lo MK, Ray AS, Mackman RL, Soloveva V, et al. Therapeutic efficacy of the small molecule GS-5734 against Ebola virus in rhesus monkeys. Nature. 2016;531:381-5.

28. Kumar GN, Jayanti VK, Johnson MK, Uchic J, Thomas S, Lee RD, et al. Metabolism and disposition of the HIV-1 protease inhibitor lopinavir (ABT-378) given in combination with ritonavir in rats, dogs, and humans. Pharmacol Res. 2004;21:1622-30.

29. Shi D, Yang J, Yang D, LeCluyse EL, Black C, You L, et al. Anti-influenza prodrug oseltamivir is activated by carboxylesterase human carboxylesterase 1 , and the activation is inhibited by antiplatelet agent clopidogrel. J Pharmacol Exp Ther. 2006;319:1477-84.

30. Jin $Q$, Feng L, Wang DD, Dai ZR, Wang P, Zou LW, et al. A two-photon ratiometric fluorescent probe for imaging carboxylesterase 2 in living cells and tissues. ACS Appl Mater Interfaces. 2015;7:28474-81.

31. Wang DD, Jin Q, Zou LW, Hou J, Lv X, Lei W, et al. A bioluminescent sensor for highly selective and sensitive detection of human carboxylesterase 1 in complex biological samples. Chem Commun. 2016;52:3183-6.

32. Zhang F, Huang J, He RJ, Wang L, Huo PC, Guan XQ, et al. Herb-drug interaction between Styrax and warfarin: molecular basis and mechanism. Phytomedicine. 2020;77:153287.

33. Fang SQ, Huang J, Zhang F, Ni HM, Chen QL, Zhu JR, et al. Pharmacokinetic interaction between a Chinese herbal formula Huosu Yangwei oral liquid and apatinib in vitro and in vivo. J Pharm Pharmacol. 2020;72:979-89.

34. Kent UM, Aviram M, Rosenblat M, Hollenberg PF. The licorice root derived isoflavan glabridin inhibits the activities of human cytochrome P450S 3A4, 2B6, and 2C9. Drug Metab Dispos. 2002;30:709-15.

35. Kim HG, Lee HS, Jeon JS, Choi YJ, Choi YJ, Yoo SY, et al. Quasi-Irreversible Inhibition of CYP2D6 by berberine. Pharmaceutics. 2020;12:916.

36. Kosaka M, Zhang D, Wong S, Yan Z. NADPH-independent inactivation of CYP2B6 and NADPH-dependent inactivation of CYP3A4/5 by PBD: potential implication for assessing covalent modulators for time-dependent inhibition. Drug Metab Dispos. 2020;48:655-61.

37. Huo $P C$, Guan $X Q$, Liu $P$, Song $Y Q$, Sun $M R$, He RJ, et al. Design, synthesis and biological evaluation of indanone-chalcone hybrids as potent and selective hCES2A inhibitors. Eur J Med Chem. 2020;209:112856.

38. Wang YQ, Weng ZM, Dou TY, Hou J, Wang DD, Ding LL, et al. Nevadensin is a naturally occurring selective inhibitor of human carboxylesterase 1. Int J Biol Macromol. 2018;120:1944-54.

39. Song YQ, Guan XQ, Weng ZM, Wang YQ, Chen J, Jin Q, et al. Discovery of a highly specific and efficacious inhibitor of human carboxylesterase 2 by large-scale screening. Int J Biol Macromol. 2019;137:261-9.

40. Song YQ, Weng ZM, Dou TY, Finel M, Wang YQ, Ding LL, et al. Inhibition of human carboxylesterases by magnolol: Kinetic analyses and mechanism. Chem Biol Interact. 2019;308:339-49.

41. Shi J, Cao B, Zha WB, Wu XL, Liu LS, Xiao WJ, et al. Pharmacokinetic interactions between 20(S)-ginsenoside Rh2 and the HIV protease inhibitor ritonavir in vitro and in vivo. Acta Pharmacol Sin. 2013;34:1349-58.

42. du Plooy $M$, Viljoen $M$, Rheeders $M$. Evidence for time-dependent interactions between ritonavir and lopinavir/ritonavir plasma levels following P-glycoprotein inhibition in Sprague-Dawley rats. Biol Pharm Bull. 2011;34:66-70.

43. Ravi PR, Vats R. Comparative pharmacokinetic evaluation of lopinavir and lopinavir-loaded solid lipid nanoparticles in hepatic impaired rat model. J Pharm Pharmacol. 2017;69:823-33.

44. Li H, Yang L, Liu FF, Ma XN, He PL, Tang W, et al. Overview of therapeutic drug research for COVID-19 in China. Acta Pharmacol Sin. 2020;41:1133-40.

45. Dunkoksung $W$, Vardhanabhuti N, Siripong $P$, Jianmongkol S. Rhinacanthin- $C$ mediated herb-drug interactions with drug transporters and phase I drugmetabolizing enzymes. Drug Metab Dispos. 2019;47:1040-9. 
46. Shaikh AS, Thomas AB, Chitlange SS. Herb-drug interaction studies of herbs used in treatment of cardiovascular disorders-A narrative review of preclinical and clinical studies. Phytother Res. 2020;34:1008-26.

47. Agarwal S, Agarwal SK. Lopinavir-ritonavir in SARS-CoV-2 infection and drug-drug interactions with cardioactive medications. Cardiovasc Drugs Ther. 2020;12:1-14.

48. Ravi PR, Vats R, Thakur R, Srivani S, Aditya N. Effect of grapefruit juice and ritonavir on pharmacokinetics of lopinavir in Wistar rats. Phytother Res. 2012;26:1490-5.

49. Riley RJ, Wilson CE. Cytochrome P450 time-dependent inhibition and induction: advances in assays, risk analysis and modelling. Expert Opin Drug Metab Toxicol. 2015;11:557-72.

50. Pasquau J, Hidalgo-Tenorio C, Montes ML, Romero-Palacios A, Vergas J, Sanjoaquín I, et al. High quality of life, treatment tolerability, safety and efficacy in HIV patients switching from triple therapy to lopinavir/ritonavir monotherapy: a randomized clinical trial. PLoS One. 2018;13:e0195068.

51. Abel S, Russell D, Taylor-Worth RJ, Ridgway CE, Muirhead GJ. Effects of CYP3A4 inhibitors on the pharmacokinetics of maraviroc in healthy volunteers. $\mathrm{Br} \mathrm{J} C$ lin Pharmacol. 2008;65:27-37.

52. Zeng $Y L$, Zeng $M Q$, Zheng $Y L$, Jiang $H M$, Lai $M$, Chen $H$, et al. Analysis of 3 death cases of critical new coronavirus pneumonia in Sichuan Province. Chin Trop Med. 2020;20:1057-61.

53. Casale M, Dattilo G, Imbalzano E, Gigliotti De Fazio M, Morabito C, Mezzetti M, et al. The thromboembolism in COVID-19: the unsolved problem. Panminerva Med. 2020;16.
54. Cremer S, Jakob C, Berkowitsch A, Borgmann S, Pilgram L, Tometten L, et al Elevated markers of thrombo-inflammatory activation predict outcome in patients with cardiovascular comorbidities and COVID-19 disease: insights from the LEOSS registry. Clin Res Cardiol. 2020;19:1-12.

55. Baranovskii DS, Klabukov ID, Krasilnikova OA, Nikogosov DA, Polekhina NV, Baranovskaia DR, et al. Prolonged prothrombin time as an early prognostic indicator of severe acute respiratory distress syndrome in patients with COVID-19 related pneumonia. Curr Med Res Opin. 2020;19:1.

56. Zhang XL, Li ZM, Ye JT, Lu J, Ye LL, Zhang CX, et al. Pharmacological and cardiovascular perspectives on the treatment of COVID-19 with chloroquine derivatives. Acta Pharmacol Sin. 2020;41:1377-86.

57. Arnold C, Markovic M, Blossey K, Wallukat G, Fischer R, Dechend R, et al. Arachidonic acid-metabolizing cytochrome $\mathrm{P} 450$ enzymes are targets of \{omega\}-3 fatty acids. J Biol Chem. 2010;285:32720-33.

58. Kroetz DL, Zeldin DC. Cytochrome P450 pathways of arachidonic acid metabolism. Curr Opin Lipidol. 2002;13:273-83.

59. Tallima $\mathrm{H}$, Ridi $\mathrm{R}$. Arachidonic acid: Physiological roles and potential health benefits-a review. J Adv Res. 2017;11:33-41.

60. Li G, Simmler C, Chen L, Nikolic D, Chen SN, Pauli GF, et al. Cytochrome P450 inhibition by three licorice species and fourteen licorice constituents. Eur J Pharm Sci. 2017;109:182-90.

61. Kent UM, Aviram M, Rosenblat M, Hollenberg PF. The licorice root derived isoflavan glabridin inhibits the activities of human cytochrome P450S 3A4, 2B6, and 2C9. Drug Metab Dispos. 2002;30:709-15. 